\title{
El Error Lingüístico, un Elemento Básico para los Especialistas del Lenguaje
}

UT. Revista de Ciències de l'Educació

Juny 2013. Pag. 29-39

ISSN 1135-1438

http://pedagogia.fcep.urv.cat/revistaut

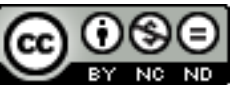

\author{
Antonio Hernández Fernández ${ }^{a}$ \\ Rebut: 19/05/2013 Acceptat: 07/06/2013
}

\section{Resumen}

Nuestro cometido inicial ha sido ofrecer al logopeda y al pedagogo una metodología mínima para distinguir los distintos tipos de errores, a fin de tomar las decisiones adecuadas para su tratamiento. Esta metodología que se ha intentado proponer constituye una guía de evaluación y de corrección que posibilite al profesor enfrentarse a los errores del aula con los conocimientos mínimos para distinguir, por ejemplo, los errores patológicos de otros tipos de errores. Nuestro trabajo constituirá la base teórica, metodológica y práctica para la futura realización de breves manuales que pondrán a disposición del logopeda y del pedagogo los conocimientos necesarios para evitar diagnósticos equivocados sobre anomalías del lenguaje.

Palabras clave: lingüística, logopeda, errores, patología, educación.

\section{The linguistic error, a basic element of language specialists}

\begin{abstract}
Our initial task was to provide the speech therapist and the teacher a minimum methodology to distinguish different types of errors, in order to make the right decisions for treatment. This methodology has tried to propose is a guide to assess and correct that enables the teacher to deal with the mistakes of the classroom with minimal knowledge to distinguish, for example, errors of other pathological types of errors. Our work will form the theoretical basis and practical methodology for the future realization of short manuals available to the speech therapist and teacher of the skills needed to avoid misdiagnosis of language abnormalities.
\end{abstract}

Keywords: linguistics, speech therapist, errors, pathology, education.

\footnotetext{
a Departamento de Pedagogía - Universidad de Jaén
} 


\section{Introducción}

Somos conscientes de que hay que tener en cuenta que el logopeda o el pedagogo no son lingüistas de profesión a menudo tienden a confundir lo que son errores patológicos, que evidencian un trastorno profundo o quizás tan sólo educacional de otros errores naturales que el niño comete simplemente porque utiliza libre y creativamente su lengua. De igual forma somos conscientes de que la investigación del error lingüístico está aún en sus comienzos. Modelos apriorísticos formalistas sobre la naturaleza del lenguaje han desdeñado durante demasiadas décadas las indagaciones sobre el "error", con la consecuencia de que no existe aún una teoría global sobre el error. A lo largo del siglo XX encontramos un extraordinario y prometedor inicio sobre el error en la obra de Frei (1929) que, sin embargo, no es continuada en las décadas siguientes. El estructuralismo y junto a él las corrientes generativistas, no ha encontrado dentro de su armazón teórica un lugar apropiado para el error y en consecuencia ha sido desterrado de sus objetivos prioritarios. Ha sido en la década de los ochenta con las obras de Fromkin (1973), Jackendoff (1990) y otros cuando el error recibe un interés más acorde con su relevancia para comprender el mecanismo de producción del lenguaje. Desde la perspectiva psicolingüística autores como Luria (1980), Garret (1981), Levelt (1989), Dell (2003), Reich (2003), entre otros, se han ocupado del error, aunque muchos de estos autores desconocen los fundamentos de la lingüística (quizás con la excepción de Luria) y por tanto, ni sus investigaciones se han aprovechado de los avances de los conocimientos lingüísticos a lo largo del siglo ni tampoco el resultado de las mismas ha influido en el pensamiento lingüístico.

\section{El error}

Casi desde el mismo momento de iniciar nuestra investigación sobre los errores tuvimos que renunciar a dos ambiciones inicialmente contempladas: apoyarnos tanto en las indagaciones como en la exposición de los resultados en un modelo lingüístico estructurado, o en su defecto crear nosotros ese modelo capaz de dar cuenta de todos los errores analizados y, al mismo tiempo, de todos los posibles errores que pudieran cometerse en la lengua. El mecanismo profundo de la producción del lenguaje está aún tan lejos de nuestros conocimientos que cualquier hipótesis sobre las causas internas responsables de la producción de un error son meras conjeturas coyunturales, difícilmente elevables a categorías capaces de articularse en un modelo holístico. Los errores son tantos y tan variados que serían necesarias aún muchas décadas de investigación para comenzar a plantearse seriamente la posibilidad de plasmar en un modelo lingüístico los mecanismos cerebrales subyacentes a la producción general del lenguaje, y en particular de los errores. Ni siquiera en el nivel metodológico hemos creído oportuno servirnos de algunos modelos muy conocidos, como por ejemplo el de Levelt (1989), quizás el más integrado y completo de los que actualmente se usan en psicología del lenguaje y logopedia, por sus evidentes 
carencias desde el punto de vista lingüístico. Investigar los errores a través de este u otro modelo hubiera sido servir al modelo, intentar encontrar los errores que encajaban con las predicciones del mismo, dejando de lado multitud de hechos lingüísticos que difícilmente tendrían cabida dentro de éste. Por otra parte, el utilizar uno de los modelos implicaría automáticamente descartar metodologías y nociones teóricas empleadas por otros modelos que creemos responden mejor a la naturaleza del error y por tanto sirven mejor para su análisis y explicación. Reflejamos en esta actitud de no atarnos a ningún modelo la posición seguida por un investigador excepcionalmente notable como es Jackendoff, quien a lo largo de sus investigaciones ha cambiado notablemente de entramado teórico, incluidos aquellos creados por él mismo. Jackendoff se plantea constantemente la necesidad de ir adecuando el modelo a los datos y a los conocimientos que estos datos nos aportan. Con todo, nuestra actitud ecléctica no ha dudado en incorporar todas aquellas nociones y planteamientos lingüísticos que desde Saussure hasta nuestros días han ido levantando la ciencia del lenguaje tal como la conocemos.

El punto inicial de nuestro trabajo fue el de revisar los planteamientos logopédicos del error y contrastarlos con los planteamientos lingüísticos del mismo. Se ha tratado de introducir el concepto de error desde el punto de vista lingüístico en el ámbito logopédico. La comunidad de logopedas es consciente de la existencia de los errores temporales, los errores de "punta de lengua", los errores de autocorrección, los errores escritos y los afásicos. Para los logopedas, y sobre todo para los psicólogos, este tipo de errores ha sido la base para la propuesta de modelos explicativos de producción lingüística. Nuestro estudio pronto nos llevó a la conclusión de que estos errores revelan gran cantidad de información sobre el lenguaje. También nos revelan que todos los modelos sobre la producción del lenguaje son parciales e incompletos. La revisión histórica que hemos realizado nos lo muestra categóricamente así: desde el mundo griego y árabe hasta la actualidad diversos autores han investigado diferentes tipos de error y han propuesto explicaciones y soluciones particulares.

\section{Marco teórico.}

Desde la gramática y la retórica tradicional hasta nuestros días son muchas y valiosas las aportaciones que pueden recogerse sobre la cuestión del error. Error, variación, falta, transgresión, solepcismo, malapropismo, lapsus, cruce, etc. son palabras que la bibliografía tradicional ha usado para identificar y analizar hechos del lenguaje comunes y literarios que forman un amplio continuum en el que se mezclan faltas contra la normativa gramatical, creaciones poéticas, detección de variedades lingüísticas de menor consideración que la variedad de la lengua estándar, etc. Todo este caudal de reflexiones sobre el "error" nos ha sido de enorme utilidad a la hora de dilucidar cuántas realidades y de qué tipo había bajo el lema común e inconcreto de error lingüístico. La incorporación de todos estos conocimientos tradicionales en un marco teórico coherente y amplio ha sido para nosotros, junto a aportaciones más recientes e innovadoras, un camino seguro para investigar el error. El marco desde el 
cual nos hemos aproximado al problema es básicamente el que Frei estableció en su "gramática de errores". En este marco teórico los conceptos de langue y parole son interdependientes, el signo lingüístico no es una realidad cerrada ni hermética sino un signo semi-abierto capaz de crecer o de saltar a otras posiciones cuando la comunicación y la carencia de otra mejor solución comunicativa así lo requiera. De igual manera, nos apartamos del signo saussureano, que sólo adquiere su valor en competencia u oposición a otros signos, y apostamos por un signo que, sin perder sus cualidades de elaboración en el seno del sistema, depende también de las influencias de los referentes con los que comunicativamente se asocia. Estas son las claves que permiten el signo metamórfico, el signo cambiante, el signo capaz de dar saltos metafóricos, metonímicos y que también resulta idóneo para realizar ampliaciones polisémicas. Además, en el modelo de la "gramática de errores" se ponen en evidencia creencias tales como la autonomía de la sintaxis o la autonomía del lexicón. Si algo deja patentemente claro los errores estudiados por nosotros es que la integración de niveles en la producción del lenguaje es un hecho indudable. Lo sintáctico puede presionar sobre lo léxico-semántico y las asociaciones semánticas permitirán cambios de sintaxis a muchas formas léxicas, tal como ya demostró Apresjan (1973). Fenómenos como la gramaticalización, la idiosincratización, idiomatización, etc. son posibles porque en el juego elástico del lenguaje, en el ir y volver de las palabras en contextos en los que hacen referencia a realidades diferentes, los hablantes pierden a menudo la noción exacta de cómo se ha de analizar el mensaje. Estas adjudicaciones y correspondencias erróneas entre formas y significados son la base de la teoría de la gramaticalización, tal como es entendida por Hopper (1991), Claudi (1991), Hünnemeyer (1991), Heine (1999), Traugott (1999), etc. Más aún, los errores lingüísticos son como los "temblores" del código o sistema, las adaptaciones necesarias del lenguaje a las nuevas necesidades comunicativas, las variaciones mediante las cuales los códigos se readaptan y reestructuran constantemente. El cambio lingüístico no es la anomalía sino la normalidad del lenguaje y todo cambio está interna y/o externamente posibilitado e individualmente iniciado. Las lenguas cambian a la larga gracias a los millones de "errores" que inevitablemente se producen en el día a día del lenguaje.

La peculiar estructura del código lingüístico, es decir la langue, junto con su empleo en el acto de habla, son los responsables del error. El error, por tanto, está potencialmente en la desigual y asimétrica estructura del código. Orden y desorden son también en el lenguaje complementarios. El desorden crea orden y el orden crea a la larga desorden. Un sistema perfecto, inmutable sería un sistema inerte, muerto. $\mathrm{Ni}$ siquiera los cristales son inertes y mucho menos una realidad compleja como el lenguaje humano. Los componentes del lenguaje, los diversos estratos y partes que han de integrarse en la producción del lenguaje lo hacen de una manera imperfecta, lo cual concede "vida" al lenguaje y permite la posibilidad de crecer, cambiar y adaptarse. Como se ha indicado, si el error no ha sido objeto de la atención que merece en la lingüística se debe a que la mayoría de las teorías lingüística del XX se han concentrado en el estudio del lenguaje como sistema estático. Ha sido sólo en la década de los ochenta cuando la teoría de la complejidad heredera de la teoría 
sistémica ha empezado a contemplar el lenguaje como un sistema autoorganizado y adaptable, capaz de cambiar o, mejor dicho, imposibilitado para no cambiar. Los avances de la ciencia neuronal nos confirman las sospechas de que diversas tareas del lenguaje se realizan en centros cerebrales diferentes que han de ser integrados en la producción e intelección del lenguaje.

Los errores lingüísticos, por tanto, nos permiten acercarnos al proceso generador del lenguaje, que según los diferentes errores que hemos estudiado parece que viene delimitado por al menos tres elementos: los contenidos nocionales (elementos conceptuales que se sitúan en el fondo u origen del proceso de la producción del lenguaje), las estructuras mecánicas que automáticamente codisponen y elicitan secuencias de elementos y las situaciones pragmáticas del discurso que exigen mayor o menor precisión informativa. Cualquier modelo que propongamos debe tener al menos tres fases: conceptual-semántica (comprende los procesos semánticos que implican la segmentación de los elementos de la conciencia en grupos proverbalizables capaces de pasar a la siguiente fase), codificación (selección de las unidades léxicas y los engramas gramaticales (verbos), que coarticulan los componentes de la oración, compleción morfológico-gramatical de las estructuras crudas para adaptarlas a la comunicación que se desea efectuar, transducción a patrones acústicos no solamente segmentales sino también suprasegmentales) y una adecuación pragmático discursiva o consideración y evaluación de información situacional y contextual que permita anticipar en las diversas fases de la elaboración o génesis del discurso la aportación informativa necesaria en cada acto comunicativo y las correcciones que eventualmente hayan de hacerse en caso de que el propio locutor se dé cuenta de que su expresión no ha sido la adecuada. Estas correcciones se manifiestan en forma de coletillas finales. Naturalmente este planteamiento, grosso modo, es sólo una generalización sobre lo que la mayoría de los lingüistas están actualmente de acuerdo: el proceso del habla, sus fases, la naturaleza de sus componentes en particular y las fases y modos de integración. No existe por ahora un acuerdo generalizado y tampoco hemos creído conveniente plantearnos el tema desde la perspectiva y objetivos de nuestro trabajo.

\section{Corpus de errores.}

Si el modelo seguido es básicamente la "gramática de errores" de Frei, adaptada y completada con las nociones que la lingüística ha desarrollado en las últimas décadas, la materia prima de nuestro trabajo han sido los "errores" que se exponen en nuestro corpus, recogidos en principio indiscriminadamente y luego con mayor precisión de búsqueda y selección. La realización de este corpus inicialmente se planteó siguiendo la metodología logopédica clásica, es decir, tratamos de inducir los errores utilizando dibujos, encuestas, trabalenguas, etc. La conclusión a la que llegamos, casi de inmediato, es que los errores provocados artificialmente eran poco significativos y en absoluto valían el esfuerzo invertido en obtenerlos. La búsqueda deliberada del error mediante provocación es, al parecer, incompatible con la propia 
naturaleza espontánea del error. Los alumnos examinados prestaban más atención y cuidaban más el lenguaje, precisamente, porque estaban realizando una tarea lingüística y eran conscientes de ello, refugiándose precisamente en los conocimientos más centrales de su sistema, en lugar de aventurarse a explorar nuevos territorios. El error surge espontáneamente y el hablante no suele ser consciente de que realiza los errores, simplemente resuelve problemas de expresión y comunicación. Los largos meses de encuestas a los alumnos aportaron un resultado extraordinariamente pobre y decepcionante, la búsqueda de errores debía de continuarse en otros terrenos y con unos medios menos ortodoxos, según los cánones de la metodología psicológica-logopédica actual. Dicho en otras palabras, el error debía ser perseguido, capturado, allí donde se diera, aunque esto no nos asegurara el control sobre edad, nivel de estudios, sexo, etc. de los hablantes que habían producido el error.

El fracaso inicial en recoger errores de manera sistemática con grupos de alumnos clasificados por edades, sexos y procedencia social y geográfica, nos llevó a la fase siguiente de recogida del corpus. El corpus de errores se había de recoger en cualquier situación o lugar y de cualquier persona que produjera el error. Este hecho sin duda alguna eliminaba la posibilidad de correlacionar tipos de errores con tipos de "sujetos", sin embargo, con la nueva "libertad" adquirida teníamos la posibilidad de ampliar mucho más rápidamente el número y la variedad de los errores. Posteriormente al analizar ciertos trabajos sobre los errores como el de Del Viso (1992) comprobamos que la misma situación en que nos encontrábamos se había dado en otros casos. Lo que perdíamos de cientificidad, al no poder utilizar los procedimientos e encuestas usuales en los estudios psicolingüísticos, lo ganábamos en frescura, originalidad y versatilidad de los errores documentados. Avanzado nuestro estudio pudimos comprobar, así mismo, que nuestra concepción inicial del error era demasiado restringida y en muchos aspectos errónea, por lo que aun si hubiéramos contado con cientos de encuestadores y miles de entrevistados los resultados tampoco hubieran sido satisfactorios. En un problema tan complejo como el de los errores, valía más dejarse llevar de la mano de lingüistas avezados como Frei o Jackendoff, quienes trabajan y reflexionan sobre errores ocasionalmente recogidos de muy diversa procedencia: calle, familia, radio, televisión, e incluso, los propios errores que uno comete y se da cuenta después de haberlos cometido. A modo de anécdota hemos de comentar nuestra sorpresa al comprobar que los modernos reality shows son una fuente magnífica para tomarle el pulso al lenguaje espontáneo y coloquial. A estos programas de televisión a menudo acuden personas sin cultura y sin un aparente miedo escénico, por lo que se expresan libremente (sobre todo las mujeres) sobre cualquier tema. Esto ofrece al lingüística investigador la capacidad de tener a mano multitud de variedades lingüísticas sin necesidad de viajar por pueblos y aldeas y permite además grabaciones que pueden ser analizadas meticulosamente. Gran cantidad de nuestros ejemplos los hemos obtenido precisamente del material así estudiado. 
Compendiando todo nuestro material, catalogándolo y analizándolo detalladamente, hemos organizado nuestro trabajo para que pueda ser una introducción o aproximación al error lingüístico con vistas a las necesidades que tienen logopedas y pedagogos de conocer y distinguir dentro de las variaciones que perciben en clase. El procedimiento seguido se ha basado en explicar en conceptos lingüísticos y gramaticales muy simples cuál es la naturaleza y los tipos de error que pueden encontrar en el entorno de su actividad profesional. Se ha tenido siempre como punto de referencia que el logopeda (es decir, un "no-lingüista" por educación) pudiera acercarse al complejo entramado del error lingüístico. La estructura del análisis que se ha seguido es: cada error se clasifica básicamente según su naturaleza en: $\mathrm{F}$ (fonológico), M (morfológico), Sx (sintáctico), Sm (semántico). El primer paso del análisis es describir superficialmente lo que "percibimos" como erróneo, es decir, describir en pocas palabras la etiología del error y su contexto histórico, geográfico, etc. cuando se conozca. La explicación de cada error se ha fundamentado en obras clave como: R.A.E. (1992, 2001), Moliner (1998), Diccionario de Dudas (1998), Diccionario del español actual (1999), Gramática didáctica del español (1999), Diccionario de español urgente (2000), Gramática española (1975), Gramática descriptiva de la lengua española (2000), etc.

El error lingüístico viene determinado por el hecho de afectar -preferentemente- a algunos elementos de la frase: verbos, pronombres, adverbios y adjetivos. Los mecanismos que se ponen en juego son: anticipación, cruce, desplazamiento, elipsis, intercambio, perseveración y transformación. El error lingüístico, en semejante medida, lo encontramos en la forma de la palabra, afectando preferentemente a verbos y adjetivos. Los mecanismos que se ponen en acción en este caso son: adición de prefijos, analogía, anticipación de morfemas, antítesis y sustitución de morfemas, cruces, desplazamientos, intercambios, movimiento incompletos de morfemas, entre otros. En menor medida, el error lingüístico afecta a la fonología de la lengua mediante los mecanismos de: adición, anticipación, cruce, desplazamiento, epéntesis, intercambio, metátesis, síncopa, elipsis y sustitución (preferentemente en consonantes). Por último, el error lingüístico afecta, en bastante menor medida, al concepto que se quiere expresar, a través de mecanismos como: colocaciones, desplazamientos semánticos, significado erróneo y falta etimología, afectando principalmente a verbos, preposiciones y sustantivos.

Todo lo anterior, sin embargo, sólo ayuda a comprender algunos aspectos del error aunque no resuelve la pregunta crucial: "¿Qué es un error?" $O$, en otras palabras, cuál es el error lingüístico genuino, distinguiéndolo de los falsos errores que sólo son variaciones sociales, geográficas, etc. Las definiciones de error de los diccionarios no son satisfactorias, ya que no abarcan todo lo analizable en esta investigación y tampoco nos discriminan los diferentes tipos de errores. Así, por ejemplo, nosotros nos hemos centrado en el error lingüístico como producto natural del lenguaje, separándolo de los errores patológicos, las trasgresiones literarias, los usos locales, las variaciones generacionales, etc. Todos estos podrían ser considerados errores en el amplio sentido de la palabra, pero son de menor interés para la lingüística. Incluso 
hemos apartado de nuestro inventario errores como el leísmo o loísmo, siempre que los produzcan personas en cuya área geográfica este fenómeno sea habitual; sí es importante si se detecta el leísmo en zonas donde no existe tal fenómeno o en personas no leístas que eventualmente cometen un leísmo, etc. Igualmente prestamos atención a qué personas no afásicas cometen errores del tipo afásico, fenómeno más corriente de lo que usualmente se cree.

Los errores en los que hemos centrado nuestra atención son los de un hablante normal, con competencia plena, sin ningún tipo de patología, que en un momento de su discurso comete algún lapsus. El error que comete esta persona ocurre por una necesidad lingüística concreta o bien por alguna particularidad del diseño propio del código lingüístico, en nuestro caso el español, que merece la pena ser investigada. El prototipo de error lingüístico viene definido por los siguientes parámetros: individualidad, competencia plena, procedencia del diseño profundo de la lengua como sistema, determinaciones del discurso como puedan ser agitación, interrupción y cualquiera de sus variantes.

\subsection{Errores fonológicos.}

Los errores fonológicos (tabla 1) son aquellos que implican a los fonemas de distintas formas. Tendremos de este modo alteraciones por adición (prótesis, epéntesis, paragoge), supresión (aféresis, síncopa, apócope), inversión (metátesis) o sustitución (antítesis).

\begin{tabular}{|l|l|l|l|}
\hline Esta masa está pegasoso. & Esta masa está pegajosa. & F & $07 / 03 / 01$ \\
\hline $\begin{array}{l}\text { Quiero una paladora corderó de acero } \\
\text { inolvidable. }\end{array}$ & $\begin{array}{l}\text { Quiero una lavadora corberó de acero } \\
\text { inoxidable. }\end{array}$ & C & $01 / 03 / 00$ \\
\hline Hago todas las mañanas puting. & Hago todas las mañanas footing. & TV & $14 / 10 / 00$ \\
\hline Estas sábanas han amarillado mucho. & Estas sábanas han amarilleado mucho. & C & $03 / 01 / 01$ \\
\hline Mi hijo es muy semental. & Mi hijo es muy sentimental. & $C$ & $24 / 08 / 00$ \\
\hline
\end{tabular}

Tabla 1. Errores fonológicos. 


\subsection{Errores morfológicos.}

Los errores morfológicos (tabla 2) son aquellos que afectan a la forma de la palabra.

\begin{tabular}{|l|l|l|l|}
\hline Estoy aburrao. & Estoy aburrido. Estoy cansado. & F & $12 / 01 / 00$ \\
\hline $\begin{array}{l}\text { ¿Cuándo se va a comprar mamá un } \\
\text { cepillador? }\end{array}$ & $\begin{array}{l}\text { ¿Cuándo se va a comprar mamá una } \\
\text { aspiradora? (cepillo + aspirador). }\end{array}$ & 09/05/00 \\
\hline $\begin{array}{l}\text { Tenemos plantados muchos alhelís. } \\
\text { Los leones son animales } \\
\text { indetomésticos. }\end{array}$ & Los leones no son animales domésticos. & $\mathrm{N}$ & $21 / 03 / 01$ \\
\hline \begin{tabular}{l} 
Esto son alumbros. \\
\hline
\end{tabular} & Esto son luces. & $\mathrm{N}$ & $21 / 03 / 01$ \\
\hline
\end{tabular}

Tabla 2. Errores morfológicos.

\subsection{Errores semánticos.}

Los errores semánticos (tabla 3) son aquellos que afectan al signo lingüístico.

\begin{tabular}{|l|l|l|l|}
\hline $\begin{array}{l}\text { ¿Quién es más rápido, el coche o la } \\
\text { bicicleta? }\end{array}$ & $\begin{array}{l}\text { ¿Qué es más rápido, el coche o la } \\
\text { bicicleta? }\end{array}$ & N & $11 / 05 / 99$ \\
\hline $\begin{array}{l}\text { El corredor entró en el quinceavo } \\
\text { puesto. }\end{array}$ & $\begin{array}{l}\text { El corredor entró en el puesto décimo } \\
\text { quinto. }\end{array}$ & R & $17 / 06 / 00$ \\
\hline $\begin{array}{l}\text { Los alumnos se dicen cansados de } \\
\text { esperar. }\end{array}$ & $\begin{array}{l}\text { Los alumnos están cansados de } \\
\text { esperar. }\end{array}$ & TV & $10 / 01 / 01$ \\
\hline ¿Qué tal estaba su español? & ¿En qué grado está su español? & C & $14 / 09 / 98$ \\
\hline Me estás revoloteando el gallinero. & Me estás revolucionando el gallinero. & C & $21 / 12 / 98$ \\
\hline
\end{tabular}

Tabla 3. Errores semánticos.

\subsection{Errores sintácticos.}

Los errores sintácticos (tabla 4) son aquellos que afectan a la coordinación y unión de palabras.

\begin{tabular}{|l|l|l|l|}
\hline $\begin{array}{l}\text { Una vez más me demuestras que } \\
\text { careces de poca educación. }\end{array}$ & $\begin{array}{l}\text { Una vez más me demuestras que } \\
\text { careces de educación. }\end{array}$ & TV & $21 / 03 / 01$ \\
\hline $\begin{array}{l}\text { Yo me parece que la solución es más } \\
\text { fácil. }\end{array}$ & $\begin{array}{l}\text { Me parece que la solución es más } \\
\text { fácil. }\end{array}$ & CLG & $24 / 01 / 01$ \\
\hline
\end{tabular}




\section{Conclusiones.}

Como conclusión de esta investigación nos queda claro que el camino que hay por delante en la investigación de los errores es muy largo, tanto para el logopeda como para el lingüista. Para la logopedia el error no debe ser sólo un indicador de la patología, sino que también debe ayudar a conocer el error normal y distinguirlo de otro tipo de error antes de proceder a realizar un diagnóstico válido o cualquier prueba o test. El error debe ser el indicador logopédico de la rehabilitación del lenguaje de una persona, debe ser en suma la llave que permite conocer el estado de las cosas en la mente del alumno. Para la lingüística, el error lingüístico debe ser una de las vías a explorar para adentrarse en los complejos procedimientos cerebrales responsables de la génesis del lenguaje. Los errores lingüísticos son las "grietas" de la estructura profunda del lenguaje, los testimonios que nos permiten constatar algunos de los más inaccesibles mecanismos internos del mismo. Los errores lingüísticos son la manifestación más palpable de los entramados cognitivos que se despliegan cuando un individuo se pone en situación comunicativa; en este sentido, una de las conclusiones más importantes de este estudio es la necesidad del error lingüístico en una lengua.

\section{Bibliografía citada.}

Agencia EFE (2000). Diccionario de español urgente. Madrid: SM.

Alcina, J. Blecua, J.M. (1975). Gramática española. Barcelona: Ariel.

Apresjan, J. D. (1973). Regular Polysemy. Linguistics, 142, págs. 5-32.

Del Viso, S. (1992). Errores espontáneos del habla y producción del lenguaje. Tesis doctoral no publicada. Universidad Complutense de Madrid.

Hopper, P.J., Traugott, E.C. (2003). Grammaticalization. Second Edition. Cambrige, UK: Cambridge University Press.

Frei, H., (1929). La grammaire des fautes. Paris: Geuther.

Fromkin, V.A. (1973). Speech errors as linguistic evidence. La Haya: Mouton.

-(1980). Errors in linguistic performance. New York: Academic Press.

Garret, M.F. (1981). Objects of psycholinguistic enquiry. Cognition, 10: 97-101.

Gómez, L. (1999). Gramática didáctica del español. Madrid: SM.

Heine, B., Traugott, E.C., eds., (1999). Approaches to Grammaticalization. Volume I. Focus on Types of Grammatical Markers. Benjamins,. Amsterdam, pp. 17-58 on theoretical and methodological issues. Amsterdam/Philadelphia: John Benjamins Publishing Company. 
Heine, B., Claudi, U., Hünnemeyer, F. (1991). Grammaticalization: A Conceptual Framework. Chicago: University of Chicago Press.

Hopper, P.J., Traugott, E.C. (2003). Grammaticalization. Second Edition. Cambrige, UK: Cambridge University Press.

Jackendoff, R. (1990). Semantic structures. Cambridge: MIT.

Jakobson, R., (1985). Ensayos de Lingüística general. Barcelona: Planeta -De Agostini.

Levelt, W.J.M. (1989). Speaking: from intention to articulation. Cambridge: MIT press.

Luria, A.R. (1968). Traumatic aphasia. Haague: Mouton. (Edic. 1970 en La Haya: Mouton.)

-(1974). Cerebro y lenguaje. Barcelona. Fontanella.

—(1980). Fundamentos de neurolingüística. Barcelona: Toray-Masson.

Moliner, M. (1998). Diccionario de uso del español. Madrid: Gredos.

R.A.E. (1992). Diccionario de la lengua española. Madrid: Espasa Calpe. (D.R.A.E. 2001: Versión electrónica 21.1.0.)

-(2000). Gramática descriptiva de la lengua española. Madrid: Espasa Calpe. (Vols. IIII)

Saussure, F. (1980). Curso de lingüística general. Madrid: Akal.

Seco, M. (1998). Diccionario de dudas y dificultades de la lengua española. Espasa Calpe: Madrid.

Seco, M., Olimpia, A., Ramos, G. (1999). Diccionario del español actual. Madrid: Aguilar.

\section{Nota biogràfica}

Antonio Hernández Fernández. Departamento de Pedagogía. Área de Didáctica y Organización Escolar. Universidad de Jaén. Campus Las Lagunillas s/n. 23071 Jaén. España. ahernand@ujaen.es

Doctor en Pedagogia y diplomado y máster en Logopedia. Entre sus obras destacar:

- Hernández, A. (2004). Los errores lingüísticos. Valencia: Nau Llibres. ISBN. 84-7642-701-8 y

- Hernández, A. (2011). Didáctica general para el grado de educación infantil. Granada: Adeo. DL. 3328-2011. ISBN. 978-84-15316-49-7. 
